\title{
Genetic elements associated with antimicrobial resistance in enteropathogenic Escherichia coli (EPEC) from Brazil
}

\author{
Isabel CA Scaletsky ${ }^{1 *}$, Tamara B Souza ${ }^{2}$, Katia RS Aranda ${ }^{1}$, Iruka N Okeke ${ }^{3}$
}

\begin{abstract}
Background: We recently observed an association of resistance with a certain enteropathogenic Escherichia coli (EPEC) serotypes and identified a conjugative plasmid, similar to plasmid pED208, that was conserved among archival $0111: \mathrm{H} 2 / \mathrm{NM}$ and $\mathrm{O} 119: \mathrm{H} 2$ strains of diverse geographical origin. In this study, we sought to determine the prevalence and distribution of this plasmid among a collection of EPEC isolates from Brazil, as well as to study the susceptibilities of these isolates to antimicrobial agents.

Results: Resistance was more commonly seen in typical EPEC than atypical strains. The most prevalent resistances were to ampicillin, tetracycline, streptomycin and the sulfonamides. Markers for the EPEC conjugative multiresistance plasmid, were detected in 21 (30\%) of typical but only $4(5 \%)$ of atypical strains ( $p=0.001$, Chisquared test). This plasmid, previously reported from only $\mathrm{O} 111$ and $\mathrm{O} 119$ strains was found in $\mathrm{O} 55$ and $\mathrm{O} 127$ strains and was associated with the presence of class 1 integrons.
\end{abstract}

Conclusion: Our data suggest a limited but expanding host range for the EPEC resistance plasmid.

\section{Background}

Enteropathogenic Escherichia coli (EPEC) is an important cause of infantile diarrhea worldwide and particularly in developing countries [1,2]. EPEC strains adhere intimately to the brush border of the intestinal epithelium and initiate a complex signaling cascade by virtue of a chromosomal pathogenicity island, the locus for enterocyte effacement (LEE) (reviewed by Clarke et al [3]). EPEC strains also carry an EPEC adherence factor (EAF) plasmid, which encodes the bundle forming pili, a plasmid-encoded regulator, and other putative virulence genes. The majority of EPEC isolates belong to classic serotypes derived from 12 classical O serogroups (O26, O55, O86, O111, O114, O119, O125, O126, O127, O128, O142, and O158) [4,5]. A robust genetic definition, and molecular and cellular detection methods, have made it possible to expand the repertoire of known EPEC lineages, identifying other EPEC that possess the LEE but do not harbor the EAF plasmid $[5,6]$. Thus, EPEC strains harboring the EAF plasmid are classified

\footnotetext{
* Correspondence: scaletsky@unifesp.br

'Departamento de Microbiologia, Immunologia e Parasitologia, Universidade Federal de São Paulo, Rua Botucatu, 862, São Paulo, 04023-062, Brazil
}

as "typical EPEC", while strains which do not harbor the EAF plasmid, are classified as atypical EPEC" [7].

For many decades, typical EPEC was the main bacterial enteropathogen in infants in Brazil. Several studies in the 1980s and early 1990s showed a high frequency of typical serotypes, particularly serotypes $\mathrm{O} 111: \mathrm{H} 2$ and O119:H6 [2,8-16]. However, some recent studies have shown a decrease in the isolation rates of these serotypes accompanied by and an apparent increase in the frequency of atypical EPEC [9,10,17-20]. Most atypical EPEC strains belong to traditional EPEC serogroups, but several strains of non-EPEC serogroups have also been identified in different epidemiological studies $[9,10,17,21]$.

Although most EPEC infections resolve without antimicrobial therapy, antimicrobials should be administered in persistent infections, where the choice of effective antimicrobials may be crucial for patient recovery and even survival [22]. In addition to a selective pressure, specifically directed towards EPEC, the persistence of resistant EPEC strains is even more likely to be related to selective pressure from antimicrobials applied at the population level. There is considerable evidence to 
suggest that young children, those most vulnerable to EPEC infection, are at risk of infection with resistant commensals, as well as pathogens, from their caregivers and household contents [23,24]. Therefore resistance genes acquired and recombined in other niches may present in EPEC strains from infants.

Many isolated enteric bacterial are known to harbor mobile elements that encode antimicrobial resistance. For example, apparently successful conserved elements have recently been described in Salmonella serovars and Yersiniae [25,26]. We recently observed an association of resistance with a certain EPEC serotypes and identified a conjugative plasmid, similar to plasmid pED208, that was conserved among archival O111:H2/NM and O119:H2 strains of diverse geographical origin [27]. However the distribution and therefore significance of this element is yet to be studied more broadly, particularly in recent isolates. In this study, we sought to determine the prevalence and distribution of this plasmid among a collection of EPEC isolates from Brazil, as well as to study the susceptibilities of these isolates to antimicrobial agents.

\section{Results and Discussion}

We assessed resistance in 149 EPEC strains (70 typical and 79 atypical) isolated from Brazilian children in previously described studies $[9,10,21]$. Typical EPEC isolates were commonly resistant to ampicillin, tetracycline, streptomycin and the sulfonamides (Table 1). All but 14 (20\%) typical strains were resistant to at least one antimicrobial and $30(43 \%)$ of the strains were resistant to three or more of the antimicrobials tested. Atypical EPEC strains were much less likely to be resistant to ampicillin, tetracycline, streptomycin and the sulfonamides, but were more likely to show resistance to trimethoprim. Although resistance to quinolones and extended-spectrum beta-lactams has emerged among enteric organisms, all the strains tested in this study were susceptible to these drugs.

EPEC strains bearing the recently reported resistance plasmid, which we sought in this study, carry at least two, and sometimes more than three, large plasmids [27]. Additionally, because the plasmid is only partially conserved, plasmid profiling cannot be used to study its distribution. Instead, we used primers that recognize traI and traC genes from the conjugative transfer region of this resistance plasmid, and the closely related plasmid pED208, to screen the recent Brazilian EPEC isolates for the presence of this element by PCR [27]. We have previously demonstrated that these primers do not produce amplicons with other known conjugative plasmids, other than those related to pED208 [27]. We additionally screened the strains for a trbC-tra $U$ region that is present in pED208 but absent from the EPEC
Table 1 Antimicrobial resistance of EPEC isolates from Brazil

\begin{tabular}{lcc}
\hline Antimicrobial & \multicolumn{2}{c}{$\mathbf{N}^{\circ}$ (\%) of resistant EPEC isolates: } \\
\cline { 2 - 3 } & $\begin{array}{c}\mathbf{t E P E C} \\
(\boldsymbol{n}=\mathbf{7 0})\end{array}$ & $\begin{array}{c}\text { aEPEC } \\
(\boldsymbol{n}=\mathbf{7 9})\end{array}$ \\
\hline Ampicillin & $42(60)$ & $19(24)$ \\
Chloramphenicol & $14(20)$ & $2(2.5)$ \\
Kanamycin & 0 & 0 \\
Sulphonamide & $44(62.8)$ & $20(25.3)$ \\
Streptomycin & $24(34.3)$ & $8(10.1)$ \\
Tetracycline & $30(42.8)$ & $8(10.1)$ \\
Trimethoprim & $1(1.4)$ & $13(16.4)$ \\
Ceftazidime & 0 & 0 \\
Ciprofloxacin & 0 & 0 \\
Lomefloxacin & 0 & 0 \\
Ofloxacin & 0 & 0 \\
Nalidixic acid & 0 & 0 \\
\hline
\end{tabular}

multiresistant plasmid. All the strains screened in this study failed to produce an amplicon with this primer pair.

As shown in Table 2, both the traI and the traC amplicons were produced in $21(30 \%)$ of typical but only $4(5 \%)$ of atypical strains $(\mathrm{p}=0.001$, Chi-squared test). Moreover, 18 (26\%) typical EPEC but only 5 (6\%) atypical EPEC produced an amplicon with at least one of the primers pairs $(\mathrm{p}=0.001)$. Of the 9 atypical EPEC that possessed the traI and/or traC marker, four belonged to O55 or O119 serogroups, which are associated with typical EPEC (see Additional file 1). These strains were negative for EAF and $b f p A$ probes, but they were positive for perA, an EAF gene [21]. Therefore, like some other atypical strains that have been described in the literature [28-30], these strains carry vestiges of the EAF plasmid.

Our data show that the EPEC resistance plasmid is found commonly in typical EPEC, and is uncommon in atypical EPEC, consistent with earlier data [27]. However, previous evaluation of the distribution of the EPEC multiresistance plasmid in a small collection of archival strains suggested that it was limited to O111:H2 and O119:H2 strains, which carry the EAF plasmid or vestiges of it [27]. In the current study, traI and traC markers from the resistance plasmid were identified in strains belonging to the serotypes O55:H6, O127:H6, and O119:H6, as well as O55 and O119 atypical strains that carry vestiges of the EAF plasmid (see Additional file 1). To determine whether this broader distribution among Brazilian isolates was a recent development, we screened 36 archival EPEC strains that were isolated in the 1970s and 1980s from children with diarrhea in São 
Table 2 Occurrence of EPEC conjugative multiresistance plasmid loci and plasmid replicons among EPEC isolates from Brazil

\begin{tabular}{ccc}
\hline Gene or Replicon & \multicolumn{2}{c}{ No. (\%) of isolates positive: } \\
\cline { 2 - 3 } & tEPEC & aEPEC \\
$(n=70)$ & $(n=79)$
\end{tabular}

Conjugative genes

tral

trac

tral+traC

$\begin{array}{ll}11(15.7) & 3(3.8) \\ 7(10) & 2(2.5) \\ 21(30) & 4(5.1)\end{array}$

Class 1 integrons

aadA1

sulll

tetA

Cat

merA

$$
\begin{gathered}
12(17.1) \\
25(35.7) \\
14(20) \\
13(18.6)
\end{gathered}
$$

$3(4.3)$

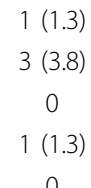

\section{Replicons}

\begin{tabular}{lcc} 
B/O & $1(1.4)$ & $1(1.3)$ \\
$\mathrm{FIC}$ & 0 & $1(1.3)$ \\
$\mathrm{A} / \mathrm{C}$ & $1(1.4)$ & $3(3.8)$ \\
$\mathrm{P}$ & $1(1.4)$ & 0 \\
$\mathrm{~T}$ & 0 & 0 \\
$\mathrm{~K} / \mathrm{B}$ & $11(15.7)$ & $15(18.9)$ \\
$\mathrm{W}$ & $3(4.3)$ & $21(26.6)$ \\
$\mathrm{FHA}$ & 0 & 0 \\
$\mathrm{FIA}$ & 0 & 0 \\
$\mathrm{FIB}$ & $32(45.7)$ & $23(29.1)$ \\
$\mathrm{Y}$ & 0 & $1(1.3)$ \\
I1 & $11(15.7)$ & $3(3.8)$ \\
Frep & $1(1.4)$ & $17(21.5)$ \\
$\mathrm{X}$ & 0 & 0 \\
HI1 & 0 & 0 \\
$\mathrm{~N}$ & 0 & 0 \\
HI2 & 0 & 0 \\
L/M & 0 & 0 \\
\hline
\end{tabular}

Paulo [12], and the plasmid was predominately found in O111:H2, O119:H6 and O142:H6 strains (data not shown), which were among the most common circulating serovars at that time [2,13,31].

Although isolates that were susceptible to all tested agents were more likely to be traI and traC negative and strains that had these markers were to a higher degree multiple resistant, in contrast to the association seen with older isolates from other geographic locations [27], we did not find that the presence of traI and traC markers in the EPEC isolates were absolutely or significantly associated with multiple resistance. The EPEC resistance plasmids of previously studied O111 and O119 strains bear class 1 integrons as well as one or more resistance genes identical to those on Salmonella enterica subsp. Typhi multiresistant plasmid pHCM1 [25]. Some typical strains and all atypical strains had fewer of these markers, even though antimicrobial resistance was just as common in these isolates (see Additional file 1). Among isolates 12 of 39 strains carrying the EPEC resistance plasmid and one of 31 strains without it had a class 1 integron $(\mathrm{p}=$ 0.0025 , Yates corrected Chi-squared test). None of the other markers screened showed significant association with the plasmid in strains. Combined with the resistance data, these findings suggest that the EPEC resistance plasmid plays less of a role in conferring resistance in these EPEC isolates, in particular atypical strains, and that there may be possible other genetic elements conferring resistance among those strains.

EPEC strains bearing the EPEC resistance plasmid carry at least two, and sometimes more than three, large plasmids $[27,30]$. We used a PCR-based replicon typing scheme to determine other possible plasmid types conferring antimicrobial resistance in the EPEC strains studied. Our results indicate that both typical and atypical strains harbor plasmid types which have been shown to carry mobile genetic elements encoding drug-resistance. In addition, significant differences in plasmid replicon content were observed between typical and atypical EPEC strains (Table 2). In particularly, the IncI1 replicon occurred significantly more often among typical strains, whereas the IncFrep replicon was observed significantly more often among atypical strains $(\mathrm{p}=0.013$ and $\mathrm{p}=0.001$, respectively) The IncT, IncFIIA, IncFIA, IncX, IncHI1, IncN, IncHI2, and IncL/M replicons were not detected in any of the strains. Among the replicon profiles identified, IncFIB occurring alone was the most common (see Additional file 1).

Antimicrobial resistance is increasing worldwide. Resistance in intestinal organisms is of interest it can compromise treatment of infections caused by pathogenic strains but also because the gut is a complex, diverse and heavily populated niche and resistant organisms there can transmit resistance genes horizontally. Many investigators have documented a high prevalence of antimicrobial resistance among EPEC strains in different parts of the world but few of these studies have been performed on recent isolates [22,32-35]. Resistance appeared at the beginning of the antibiotic era and epidemiological data suggests that its prevalence is associated with the 1970s and 1980s and diversity of antimicrobial use [33,35]. The genetic basis for this resistance and the evolutionary consequences are rarely studied.

\section{Conclusion}

Our data show that the EPEC resistance plasmid is found commonly in typical EPEC, and is uncommon in 
atypical EPEC, consistent with earlier data. However, previous evaluation of the distribution of the EPEC multiresistance plasmid in a small collection of archival strains suggested that it was limited to $\mathrm{O} 111: \mathrm{H} 2$ and O119:H2 strains, which carry the EAF plasmid or vestiges of it. In this study, the host range of the EPEC resistance plasmid, although still largely restricted to typical EPEC, was seen to be greater in recent isolates.

\section{Methods}

\section{Bacterial strains}

The 149 strains examined in this report were isolated between 1997-1999 during an epidemiological study of acute diarrhea in children $<2$ years of age conducted in different regions of Brazil and between 2002 to 2003 from children $<5$ years of age with diarrhea in São Paulo $[9,10,21]$. These strains were identified by hybridization with eae and/or EAF probe sequences and serotyped. Most of these EPEC strains had also been characterized by the presence of LEE-associated DNA sequences, and $b f p A$ and per $A$ sequences, and adherence to HEp-2 cells [21].

\section{Preparation of bacterial DNA and PCR amplification for} detection of the EPEC conjugative multiresistance plasmid, class 1 integron and plasmidreplicons

The bacterial DNA was extracted from a single colony on a LB agar plate. The bacteria were suspended in 500 $\mu \mathrm{l}$ of $1 \mathrm{X}$ phosphate-buffered saline ( $\mathrm{pH}$ 7.4) solution, boiled for $10 \mathrm{~min}$, and centrifuged. Two microliters of the resulting supernatant was used as template DNA in $25 \mu \mathrm{l}$ of the PCR mixture. Each reaction mixture contained $0.4 \mathrm{mM}$ deoxynucleoside triphosphates, $1 \mathrm{U}$ of Taq polymerase, $1 \times$ Taq reaction buffer, and $2 \mu \mathrm{M}$ of each of the primers. Primer sequences and PCR conditions used were as previously published [27]. Strains were screened for the presence of the plasmid pMB80 by PCR using primers complementary to internal regions of the traI and $\operatorname{traC}$ genes that are conserved between the MB80 tra system and the closely related plasmid pED208, as well as one primer pair whose product straddles traU and trbC in pED280 in a region not conserved in pMB80 [27]. Strains were screened for class 1 integrons using primers designed by Levesque et $\mathrm{al}[36]$ as well as for the presence of sulII gene (conferring sulphonamide resistance), tet $A$ gene (tetracycline resistance), trimetroprim resistance gene, cat (kanamycin resistance), $\operatorname{str} A B$ (streptomycin resistance), and a mer operon (mercury resistance) using primers originally designed for the Salmonella enteric serovar Typhi multiresistant plasmid, pHCM1 [25]. EPEC strains were examined for the presence of 18 plasmid replicons using three multiplex panels described by Johnson et al. [37]. PCR products were visualized on a $1.5 \%$ agarose gel stained with ethidium bromide and visualized under UV transillumination.

\section{Antimicrobial susceptibility test}

All strains were tested for their susceptibility to 12 antimicrobial agents commonly used in Brazil $[38,39]$ by the broth microdilution method according to the Clinical Laboratory Standards Institute [40]. Minimal inhibition concentration (MIC) breakpoint levels and concentration of each antimicrobial were based on those specified by the CLSI. Intermediately susceptible strains were recorded as being susceptible. E. coli strain 25922 (ATCC) was used as the reference strain. All strains were examined for resistance to ampicillin, ceftazidime, ciprofloxacin, chloramphenicol, kanamycin, lomefloxacin, ofloxacin, streptomycin, nalidixic acid, sulfonamide, tetracycline, and trimethropin.

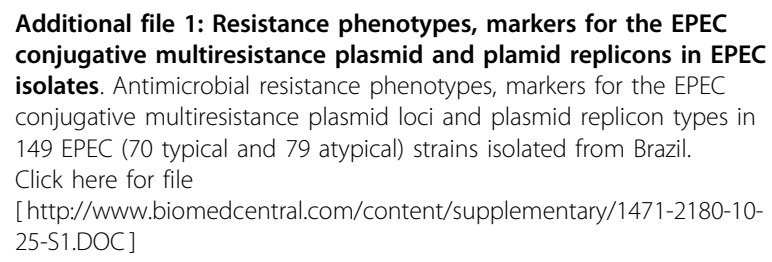

\section{Acknowledgements}

This work was supported by Branco Weiss Fellowship to INO, Fundação de Amparo a Pesquisa de São Paulo (Fapesp), and Conselho Nacional de Desenvolvimento Científico e Tecnológico (CNPq). Other support currently held by the authors includes NSF grant to INO (RUI\#0516591),

\section{Author details}

'Departamento de Microbiologia, Immunologia e Parasitologia, Universidade Federal de São Paulo, Rua Botucatu, 862, São Paulo, 04023-062, Brazil. ${ }^{2}$ Departamento de Pediatria, Universidade Federal de São Paulo, Rua Botucatu 598, São Paulo, 04023-062, Brazil. ³ Department of Biology, Haverford College, 370 Lancaster Avenue, Haverford PA, 19041, USA.

\section{Authors' contributions}

ICAS and INO conceived the study and wrote the paper. TBS and KRSA performed the laboratory studies. All authors read and approved the final manuscript.

Received: 16 April 2009

Accepted: 27 January 2010 Published: 27 January 2010

\section{References}

1. Nataro JP, Kaper JB: Diarrheagenic Escherichia coli. Clin Microbiol Rev 1998, 11:142-201.

2. Gomes TAT, Griffin PM, Ivey C, Trabulsi LR, Ramos SRTS: EPEC infections in Sao Paulo. Revista de Microbiologia. J Brazil Soc Microbiol 1996, 27:25-33.

3. Clarke SC, Haigh RD, Freestone PPE, Williams PH: Virulence of enteropathogenic Escherichia coli, a global pathogen. Clin Microbiol Rev 2003, 16:365-378.

4. Reid S, Herbelin C, Bumbaugh A, Selander R, Whittam T: Parallel evolution of virulence in pathogenic Escherichia coli. Nature 2000, 406:64-67.

5. Wirth T, Falush D, Lan R, Colles F, Mensa P, Wieler LH, Karch H, Reeves PR, Maiden MC, Ochman H, Achtman M: Sex and virulence in Escherichia coli: an evolutionary perspective. Mol Microbiol 2006, 60:1136-1151.

6. Lacher DW, Steinsland H, Blank TE, Donnenberg MS, Whittam TS: Molecular evolution of typical enteropathogenic Escherichia coli: Clonal analysis by 
multilocus sequence typing and virulence gene allelic profiling. $J$ Bacteriol 2007, 189:342-350.

7. Trabulsi LR, Keller R, Tardelli Gomes TA: Typical and atypical enteropathogenic Escherichia coli. Emerg Infect Dis 2002, 8:508-513.

8. Rosa AC, Mariano AT, Pereira AM, Tibana A, Gomes TA, Andrade JR: Enteropathogenicity markers in Escherichia coli isolated from infants with acute diarrhoea and healthy controls in Rio de Janeiro, Brazil. J Med Microbiol 1998, 47:781-790.

9. Scaletsky ICA, Pedroso MZ, Oliva CAG, Carvalho RLB, Morais MB, FagundesNeto U: A localized adherence-like pattern as a second pattern of adherence of classic enteropathogenic Escherichia coli to HEp-2 cells that is associated with acute infantile diarrhea. Infect Immun 1999, 67:3410-3415.

10. Scaletsky ICA, Fabbricotti SH, Silva SO, Morais MB, Fagundes-Neto U: HEp-2adherent Escherichia coli strains associated with acute infantile diarrhea, São Paulo, Brazil. Emerg Infect Dis 2002, 8:855-858.

11. Campos LC, Franzolin MR, Trabulsi LR: Diarrheagenic Escherichia coli categories among the traditional enteropathogenic $E$. coli $O$ serogroupsa review. Mem Inst Oswaldo Cruz 2004, 99:545-552.

12. Gomes TA, Vieira MA, Wachsmuth IK, Blake PA, Trabulsi LR: Serotypespecific prevalence of Escherichia coli strains with EPEC adherence factor genes in infants with and without diarrhea in São Paulo, Brazil. J Infect Dis 1989, 160:131-135.

13. Magalhaes M, Amorim RJ, Takeda Y, Tsukamoto T, Antas MG, Tateno S: Localized, diffuse, and aggregative-adhering Escherichia coli from infants with acute diarrhea and matched-controls. Mem Inst Oswaldo Cruz 1992, 87:93-97.

14. Tsukamoto T, Takeda Y: Incidence and prevalence of serotypes of enteroaggregative Escherichia coli from diarrheal patients in Brazil, Myanmar and Japan. Kansenshogaku Zasshi 1993, 67:289-294

15. Stewien KE, Mós EN, Yanaguita RM, Jerez JA, Durigon EL, Hársi CM, Tanaka H, Moraes RM, Silva LA, Santos MA: Viral, bacterial and parasitic pathogens associated with severe diarrhoea in the city of São Paulo, Brazil. J Diarrhoeal Dis Res 1993, 11:148-152.

16. Gomes TA, Rassi V, MacDonald KL, Ramos SRTS, Trabulsi LR, Vieira MAM, Guth BEC, Candeias JAN, Ivey C, Toledo MRF, Blake PA: Enteropathogens associated with acute diarrheal disease in urban infants in São Paulo, Brazil. J Infect Dis 1991, 164:331-337.

17. Nguyen RN, Taylor LS, Tauschek M, Robins-Browne RM: Atypical enteropathogenic Escherichia coli infection and prolonged diarrhea in children. Emerg Infect Dis 2006, 12:597-603.

18. Orlandi PP, Magalhães GF, Matos NB, Silva T, Penatti M, Nogueira PA, Silva LH: Etiology of diarrheal infections in children of Porto Velho (Rondonia, Western Amazon region, Brazil. Braz J Med Biol Res 2006, 39:507-517.

19. Afset JE, Bevanger L, Romundstad P, Bergh K: Association of atypical enteropathogenic Escherichia coli (EPEC) with prolonged diarrhoea. J Med Microbiol 2004, 53:1137-1144.

20. Afset JE, Bergh $\mathrm{K}$, Bevanger L: High prevalence of atypical enteropathogenic Escherichia coli (EPEC) in Norwegian children with diarrhoea. J Med Microbiol 2003, 52:1015-1019.

21. Dulguer MV, Fabbricotti SH, Bando SY, Moreira-Filho CA, Fagundes-Neto U, Scaletsky IC: Atypical enteropathogenic Escherichia coli strains: phenotypic and genetic profiling reveals a strong association between enteroaggregative $E$. coli heat-stable enterotoxin and diarrhea. $J$ Infect Dis 2003, 188:1685-1694.

22. Senerwa D, Mutanda LN, Gathuma JM, Olsvik O: Antimicrobial resistance of enteropathogenic Escherichia coli strains from a nosocomial outbreak in Kenya. Apmis 1991, 99:728-734.

23. Lietzau S, Raum E, von Baum H, Marre R, Brenner H: Household contacts were key factor for children's colonization with resistant Escherichia coli in community setting. J Clin Epidemiol 2007, 60:1149-1155.

24. Zaidi MB, Zamora E, Diaz P, Tollefson L, Fedorka-Cray PJ, Headrick ML: Risk factors for fecal quinolone-resistant Escherichia coli in Mexican children. Antimicrob Agents Chemother 2003, 47:1999-2001.

25. Wain J, Diem Nga LT, Kidgell C, James K, Fortunate S, Song Diep T, Ali T, Gaora PO, Parry C, Parkhill J, Farrar J, White NJ, Dougan G: Molecular analysis of incHI1 antimicrobial resistance plasmids from Salmonella serovar Typhi strains associated with typhoid fever. Antimicrob Agents Chemother 2003, 47:2732-2739.
26. Welch TJ, Fricke WF, McDermott PF, White DG, Rosso ML, Rasko DA, Mammel MK, Eppinger M, Rosovitz MJ, Wagner D, Rahalison L, Leclerc JE, Hinshaw JM, Lindler LE, Cebula TA, Carniel E, Ravel J: Multiple antimicrobial resistance in plague: an emerging public health risk. PLOS ONE 2007, 2: e309.

27. Nwaneshiudu Al, Mucci T, Pickard DJ, Okeke IN: A second large plasmid encodes conjugative transfer and antimicrobial resistance inO119: $\mathrm{H} 2$ and some typical 0111 enteropathogenic Escherichia coli strains. J Bacteriol 2007, 189:6074-6079.

28. Bortolini M, Trabulsi L, Keller R, Frankel G, Sperandio V: Lack of expression of bundle-forming pili in some clinical isolates of enteropathogenic Escherichia coli (EPEC) is due to a conserved large deletion in the bfp operon. FEMS Microbiol Lett 1999, 169-174.

29. Okeke IN, Borneman JA, Shin S, Mellies JL, Quinn LE, Kaper JB: Comparative sequence analysis of the plasmid-encoded regulator of enteropathogenic Escherichia coli strains. Infect Immun 2001, 69:5553-5564.

30. Fernandes R, Ramos S, Rassi V, Blake P, Gomes T: Use of plasmid profiles to differentiate strains within specific serotypes of classical enteropathogenic Escherichia coli. Braz J Med Biol Res 1992, 25:667-672.

31. Lim YS, Ngan CC, Tay L: Enteropathogenic Escherichia coli as a cause of diarrhoea among children in Singapore. J Trop Med Hyg 1992, 95:339-342.

32. Vila J, Vargas M, Casals C, Urassa H, Mshinda H, Schellemberg D, Gascon J. Antimicrobial resistance of diarrheagenic Escherichia coli isolated from children under the age of 5 years from Ifakara, Tanzania. Antimicrob Agents Chemother 1999, 43:3022-3024.

33. Moyenuddin M, Wachsmuth IK, Moseley SL, Bopp CA, Blake PA: Serotype, antimicrobial resistance, and adherence properties of Escherichia coli strains associated with outbreaks of diarrheal illness in children in the United States. J Clin Microbiol 1989, 27:2234-2239.

34. Gross RJ, Ward LR, Threlfall EJ, King H, Rowe B: Drugresistance among infantile enteropathogenic Escherichia coli strains isolated in the United Kingdom. Br Med J (Clin Res Ed) 1982, 285:472-473.

35. Slocombe B, Sutherland R: Transferable antibioticresistance in enteropathogenic Escherichia coli between 1948 and 1968. Antimicrob Agents Chemother 1973, 4:459-466.

36. Lévesque C, Piché L, Larose C, Roy PH: PCR mapping of integrons reveals several novel combinations of resistance genes. Antimicrob Agents Chemother 1995, 39:185-191.

37. Johnson TJ, Wannemuehler YM, Johnson SJ, Logue CM, White DG, Doetkott C, Nolan LK: Plasmid replicon typing of commensal and pathogenic Escherichia coli isolates. Appl Environ Microbiol 2007, 73:1976-1983.

38. Berquo LS, Barros AJ, Lima RC, Bertoldi AD: Use of drugs to treat respiratory tract infections in the community. Rev Saude Publica 2004, 38:358-364.

39. Berquo LS, Barros AJ, Lima RC, Bertoldi AD: Use of antimicrobial drugs in an urban population. Rev Saude Publica 2004, 38:239-246.

40. National Committee for Clinical Laboratory Standards: Performance standards for antimicrobial disk susceptibility tests. National Committee for Clinical Laboratory Standards, Villanova, PA, 82003.

doi:10.1186/1471-2180-10-25

Cite this article as: Scaletsky et al: Genetic elements associated with antimicrobial resistance in enteropathogenic Escherichia coli (EPEC) from Brazil. BMC Microbiology 2010 10:25. 